\title{
Regularization of gauge theory on noncommutative
} $\mathbb{R}^{4}$

\author{
Wolfgang Behr* \\ MPI fuer Physik, Germany \\ E-mail: behr@theorie.physik. uni-muenchen. de
}

\begin{abstract}
In gauge theory on noncommutative spacetime with constant commutator, the infinities of commutative gauge theory persist and new infinities (the famous IR/UV-mixing) show up. To deal with these, a consistent way to regularize noncommutative QFT is needed. For the regularization we will use a matrix model whose ground state is the product of two fuzzy spheeres, the fluctuations around this ground state producing the gauge theory. This gauge theory is completely well defined and finite. In a double scaling limit we will blow up the fuzzy spheres at their north poles, mapping the gauge theory on the spheres to the gauge theory on noncommutative $\mathbb{R}^{4}$, and thereby providing it with the desired reularization. Further we were able to match certain sectors of the instanton solutions of the regularized theory with known fluxon-solutions on noncommuative $\mathbb{R}^{4}$. The talk is based on joint work with Frank Meyer and Harold Steinacker [1].
\end{abstract}

International Europhysics Conference on High Energy Physics

July 21st - 27th 2005

Lisboa, Portugal

* Speaker. 
One of the motivations for introducing noncommutative structures in physics was to get a better control of the infi nities in quantum fi eld theory. At least for the canonical case of constant commutator between the coordinates, this hope was not fulfi lled. The infi nities of the commutative theory persist, and even new phenomena like the IR/UV-mixing were found. To handle these problems, a consistent way to regularize noncommutative QFTs is needed. In this talk we will present such a regularization of noncommutative gauge theory on $\mathbb{R}_{\theta}^{4}$.

In the canonical case, the noncommutative space $\mathbb{R}_{\theta}^{4}$ is generated by coordinates with commutation relations

$$
\left[x_{i}, x_{j}\right]=i \theta_{i j} \text { with } \theta_{i j} \in \mathbb{R}
$$

By suitable rotations and complexifi cation, this can always be brought to the form of two Heisenberg algebras

$$
\left[x_{L}^{+}, x_{L}^{-}\right]=\theta, \quad\left[x_{R}^{+}, x_{R}^{-}\right]=\theta \text { and }\left[x_{L}^{ \pm}, x_{R}^{ \pm}\right]=0,
$$

which can now be represented on the usual Fock space. Derivatives are internal operations on this space, i.e. $\partial_{i} \hat{=}-\frac{i}{\theta}\left[x_{i}, \cdot\right]$. Gauge theory can be formulated as a matrix model with infi ntedimensional matrices $X$ and an action

$$
S=-\frac{(2 \pi)^{2}}{2 g^{2} \theta^{2}} \operatorname{tr}\left(\left[X_{i}, X_{j}\right]-i \theta_{i j}\right)^{2} .
$$

The ground state of such a theory obviously is $\mathbb{R}_{\theta}^{4}$, and fluctuations $A_{i}$ around this ground state will produce a gauge theory with covariant coordinates $X_{i}=x_{i}+A_{i}$ transforming as

$$
\begin{aligned}
X_{i} & \rightarrow U X_{i} U^{\dagger} \\
A_{i} & \rightarrow U\left[x_{i}, U^{\dagger}\right]+U A_{i} U^{\dagger}
\end{aligned}
$$

under unitary gauge transformations $U$. The fi eld strength is defi ned as

$$
i F_{i j}=\left[X_{i}, X_{j}\right]-i \theta_{i j}=\left[x_{i}, A_{j}\right]-\left[x_{j}, A_{i}\right]+\left[A_{i}, A_{j}\right]
$$

It is well known that this theory is not free of infi nities, but there is also another problem linked to the infi nite-dimensional representation of the space: it contains sectors for gauge groups $U(N)$ of arbitrary rank. If $x^{i}$ is a ground state of (3), then $x_{i} \otimes 1_{N \times N}$ is a ground state as well! And the related covariant coordinates $X_{i}=x_{i} \otimes 1_{N \times N}+A_{i, a} T^{a}$ with $T^{a}$ the generators of $u(N)$ will therefore produce a $U(N)$ gauge theory. We will see that this problem is absent in our regularized theory.

For the regularization we will follow the ideas of [2], using a spacetime generated by two sets of fuzzy spheres. Such a fuzzy sphere is a $M$-dimensional (and therfore fi nite) representation of $s u(2)$, with generators fulfi lling

$$
\left[\lambda_{i}, \lambda_{j}\right]=i \varepsilon_{i j k} \lambda_{k} \text { and } \lambda_{1}^{2}+\lambda_{2}^{2}+\lambda_{3}^{2}=\frac{M^{2}-1}{4} .
$$

The coordinates are linked to the generators by $x_{i}=\frac{2 R}{\sqrt{M^{2}-1}} \lambda$ and the tangential derivatives are again inner, i.e. $J_{i}=\left[\lambda_{i}, \cdot\right]$. The four-dimensional space is then generated by two sets $\lambda_{i L}$ and $\lambda_{i R}$, which are now $M^{2}$-dimensional matrices. The gauge theory can be introduced in much the same way as in the canonical case by setting a matrix action

$$
S=\frac{8 \pi^{2}}{M^{2}} \operatorname{tr}\left(\left(i\left[B_{i L / R}, B_{j L / R}\right]+\varepsilon_{i j k} B_{k L / R}\right)^{2}-\left[B_{i L}, B_{j R}\right]^{2}+V(B)\right),
$$


where the potential $V(B)=2\left(B_{i L} B_{i L}-\frac{M^{2}-1}{4}\right)^{2}+2\left(B_{i R} B_{i R}-\frac{M^{2}-1}{4}\right)^{2}$ stabilizes the radii of the spheres. The ground states are obviously $\lambda_{i L}=\lambda_{i} \otimes 1$ and $\lambda_{i R}=1 \otimes \lambda_{i}$, and the covariant coordinates $B_{i L / R}=\lambda_{i L / R}+A_{i L / R}$ again transform as

$$
\begin{aligned}
& B_{\mu} \rightarrow U B_{\mu} U \\
& A_{\mu} \rightarrow U\left[\lambda_{\mu}, U\right]+U A_{\mu} U
\end{aligned}
$$

producing a gauge theory with fi eld strength

$$
F_{i L j L}=\left[\lambda_{i L}, A_{j L}\right]-\left[\lambda_{j L}, A_{i L}\right]+\left[A_{i L}, A_{j L}\right]-i \varepsilon_{i j k} A_{k L}
$$

Quantization can be performed by doing a path integral over the matrix entries as

$$
Z[J]=\int d B_{\mu} e^{-S\left[B_{\mu}\right]+\operatorname{tr} B_{\mu} J_{\mu}}
$$

Note that everything is fi nite because the trace is over a fi nite dimensional space. Also, the rank of the gauge group is fi xed (in our case to $N=1$ ), because we are using $M$-dimensional matrices. To construct a $U(N)$ gauge theory, we have to use $N M^{2}$-dimensional matrices, the potential $V$ singling out $\lambda_{\mu} \otimes 1_{N \times N}$ as the ground state.

For the coordinates, the limit to $\mathbb{R}_{\theta}^{4}$ can be done by letting $M$ go to infi nity and at the same time blowing up the spheres around the north poles by setting $R^{2}=M \theta / 2$. Then the coordinates fulfi 1

$$
\left[x_{1}, x_{2}\right]=i \frac{2 R}{N} \sqrt{R^{2}-x_{1}^{2}-x_{2}^{2}}=i \theta+O(1 / N) .
$$

The same can be done for the covariant coordinates, setting

$$
\sqrt{\frac{2 \theta}{M}} B_{1,2 /, L} \rightarrow X_{1,2} \text { and } \sqrt{\frac{2 \theta}{M}} B_{1,2 /, R} \rightarrow X_{3,4}
$$

and thereby mapping the gauge theory on the fuzzy spheres to the one on $\mathbb{R}_{\theta}^{4}$. To confi rm this also in the nonperturbative regime, we constructed a part of the known instanton solutions on $\mathbb{R}_{\theta}^{4}$ from instantons on the fuzzy spheres. Surprisingly, the regularization works as a superselection rule on the instanton charge. On $\mathbb{R}_{\theta}^{4}$, instantons with charge $k$ can simply be written as

$$
X_{\mu}=\left(\begin{array}{cc}
\operatorname{diag}\left(c_{1, \mu}, \ldots, c_{k, \mu}\right) & 0 \\
0 & x_{\mu}
\end{array}\right)
$$

We can mimick this construction on the spheres by setting

$$
B_{\mu}=\left(\begin{array}{cc}
\operatorname{diag}\left(d_{1, \mu}, \ldots, d_{k, \mu}\right) & 0 \\
0 & \lambda_{\mu}
\end{array}\right)
$$

but here the dimension of the $B_{\mu}$ is fixed to $M^{2}$. Of course we can use representations $\lambda_{\mu}$ of dimension $M^{\prime}=(M-l)(M-m)$, but this means that the only fi nite instantons allowed are those with $l=-m$ and instanton charge $k=l^{2}$. 


\section{References}

[1] W. Behr, F. Meyer and H. Steinacker, Gauge theory on fuzzy $S^{2} \times S^{2}$ and regularization on noncommutative $\mathbb{R}^{4}$, JHEP 0507:040,2005, [hep-th/0503041]

[2] Harold Steinacker, Quantized gauge theory on the fuzzy sphere as random matrix model,Nucl.Phys.B679:66-98,2004, [hep-th/0307075] 